\title{
Flexural Analysis of Kirchhoff plates on Winkler foundations using finite Fourier sine integral transform method
}

\author{
Charles C. Ike
}

Email: ikecc2007@yahoo.com

\begin{abstract}
In this work, the Fourier sine transform method has been applied to solve the flexural problem of rectangular Kirchhoff plates resting on Winkler foundations for the case of simply supported edges and transverse distributed loads. The Fourier sine transformation was applied to the governing partial differential equation, and the boundary value problem simplified to an algebraic problem. By inversion, solutions were obtained for the general case of arbitrary distributed load and for particular cases of point load, patch load, sinusoidal load, uniform load and linearly distributed loads. It was found that the solutions obtained were exact solutions, and were exactly identical with the solutions obtained in literature using Navier's double trigonometric series methods. The effectiveness of the Fourier sine transform method was thus illustrated.
\end{abstract}

Keywords: Finite Fourier Sine Transform Method, Kirchhoff Plate, Winkler Foundation, Navier'S Double Trigonometric Series Method, Boundary Value Problem.

\section{INTRODUCTION}

Flexural problems of plates resting on elastic foundations and carrying transverse distributed loads are common in geotechnical and structural engineering. They are encountered in the analysis of foundation structures such as column footings, combined footings, and plate or raft foundations. They are also encountered in problems of structural analysis that are described by mathematical equations that are analogues of the fourth order partial differential equation of the Kirchhoff plate on Winkler foundation problem. Such problems of soil structure interaction are described by incorporating the soil interaction/reaction model into the governing equations of the plate structure in a variational formulation or equilibrium formulation.

Many theories exist for the description of the flexural behaviour of plates. They include: Lagrange plate theory, Germain theory, Kirchhoff plate theory, Love plate theory, von Karman theory, Reissner [1, 2] plate theory, Mindlin [3] plate theory, Shimpi [4] refined plate theory, Reddy [5] plate theory and other variants of the refined plate theories and shear deformation plate theories. Kirchhoff's plate theory, otherwise called the classical thin plate theory or the Kirchhoff - Love plate theory is adopted in this paper. The theory is ideally suitable for thin plate for which the thickness $h$ to least governing span $a$ ratio is less than $1 / 20$.

The fundamental hypothesis of the theory are:

(i)Straight lines that are normal to the middle surface of the plate before bending remain straight after bending deformation.
(ii)Straight lines normal to the plate middle surface before flexural deformation remain normal to the middle surface after flexural deformation.

(iii)The thickness of the plate does not change during bending deformation.

The Kirchhoff plate theory is a two dimensional approximation of the classical mathematical theory of elasticity in three dimensional space applied to the problem of plates in order to determine the stress and displacement fields in the plate, considered thin, and subjected to forces and moments under different restraint conditions. It can be considered a two dimensional extension of the one dimensional Euler - Bernoulli beam theory. It assumes that a middle plane surface assumed neutral during flexural deformations can be used to represent a three dimensional plate in two dimensional domain [6]. The obvious advantages of the Kirchhoff plate theory are:

(i)The three dimensional problem of plate is simplified by reduction to a two dimensional problem.

(ii)The bending and stretching behavious are uncoupled.

(iii)The linearity property of the governing partial differential equation, renders the equations solvable within the plate domain using techniques for solving linear partial differential equations.

(iv)Stresses can be found from the displacements using the stress displacement relations.

(v)It is commonly applied to plate problems that are classified as thin.

The most significant defect of the theory is the disregard of the transverse shear deformation and its inability to correctly 
analyse plate bending problems in which transverse shear deformation plays a significant role.

The interaction of the soil on the interfacing foundation structure, is represented by the soil reactive pressure distribution [7]. Many mathematical expressions exist for describing the soil interaction on the interfacing foundation structure. The elastic foundation models are broadly categorized as discrete foundation models, simplified elastic continuum models, and elastic continuum models $[8,9]$.

In discrete parameter models, the elastic foundation, which is a continuous body is discretized and replaced with a set of closely spaced, individual elastic springs that may or may not be connected or coupled to one another.

In continuum models, the mathematical theory of elasticity in three dimensional space variable is used as an analytical and theoretical framework to determine complex analytical expressions that describe the soil reactions on the interfacing foundation structure. Simplified elastic continuum foundation models are simplifications of the rigorous theory of elasticity formulation for the soil reactions; obtained by the use of simplifying assumptions in respect of stresses or deformations. Discrete parameter foundation models include:

(i)Winkler [10] foundation model.

(ii)Pasternak [11] foundation model.

(iii)Filonenko - Borodich [12] foundation model.

(iv)Hetenyi [13] model.

(v)Generalized two parameter foundation model.

(vi)Generalized $n$ parameter foundation model.

The simplest soil structure interaction model is provided by the classical Winkler foundation, which idealises the foundation using a mechanical analogy made of a single bed of closely spaced, independent vertical elastic springs, that do not interact with one another. In the Winkler foundation model, the soil reaction on the plate soil - interface is directly proportional to the deformation of the plate at that point. This yields the mathematical idealization

$$
p(x, y)=k_{s} w(x, y)
$$

where $p(x, y)$ is the soil reactive pressure distribution at an arbitrary point $(x, y)$ on the plate - soil interface area, $w(x, y)$ is the corresponding vertical deformation and $k_{s}$ is a proportionality constant, representing the contact pressure per unit deformation. $k_{s}$ is commonly called the Winkler coefficient or coefficient of subgrade modulus. In the Winkler model also called, a one parameter model, $k_{s}$ is the only foundation parameter characterising the elastic response of the soil. Winkler foundation model suffers the defect of discontinuity of deformation at the edges of the plate, since there occurs only vertical deformation of only those springs that are located under the loaded plate.

Another shortcoming of the Winkler foundation model is that the vertical deformation at any point is independent of the vertical deformation of other adjoining points, in contradiction to the theory of elasticity. These shortcomings have resulted in the development of other discrete parameter models, which account for shear interaction [11]. However, the simple nature of the Winkler model equation and its extensive use has ensured its widespread usage till today.

The Filonenko - Borodich, Pasternak and generalised two parameter foundation models are discrete parameter foundation models where the soil reaction pressure $p(x, y)$ is given in general by [11. 12]:

$$
p(x, y)=k_{1} w(x, y)-k_{2} \nabla^{2} w(x, y)
$$

where $k_{1}$ and $k_{2}$ are the two discrete parameters of the model, and $\nabla^{2}$ is the Laplacian operator in two dimensional Cartesian coordinates, given by:

$\nabla^{2}=\frac{\partial^{2}}{\partial x^{2}}+\frac{\partial^{2}}{\partial y^{2}}$

In the Kerr model, a shear layer is introduced in the conventional Winkler bed of elastic springs. The shear layer separates the elastic bed into two different beds characterized by two different spring constants; $k_{1}$ for the first layer interfacing the plate and $k_{2}$ for the second layer that is in contact with a rigid base. The governing equation for the Kerr [14] formulation model is given by the differential equation:

$$
\left(1+\frac{k_{2}}{k_{1}}\right) p=\frac{G}{k_{1}} \nabla^{2} p+k_{2} w-G \nabla^{2} w
$$

where $k_{1}$ is the spring constant of the first bed of springs, $k_{2}$ the spring constant of the second bed of elastic springs, $G$ the shear modulus of the shear layer which separates the first and second layers and $w(x, y)$ is the deflection of the first layer.

\section{Research aim and objectives}

The aim of this research is to use the finite Fourier sine transform method to solve the problem of flexure of simply supported Kirchhoff plate resting on Winkler foundation. The specific objectives are:

(i)to apply the Fourier sine transform method to obtain the general solution to the flexural problem of simply supported Kirchhoff plate resting on Winkler foundation for the case of general distributed transverse load.

(ii)to transform the boundary value problem of Kirchhoff plate on Winkler foundation under arbitrary distribution of transverse load to an algebraic problem using Fourier sine transformation.

(iii)to solve the resulting algebraic equation to obtain solutions for the general case of distributed transverse load on the Kirchhoff plate on Winkler foundation.

(iv)to obtain solutions for the flexural problem of Kirchhoff plate on Winkler foundation for particular types of transverse load, namely:

(a) point load applied at a known point $(\mathrm{x} 0, \mathrm{y} 0)$ on the plate region

(b)bisinusoidal distribution of load over the entire plate region.

(c)uniformly distributed transverse load on the entire plate region and

(d)linearly distributed transverse load over the entire plate region.

\section{Theoretical framework/governing equation}

A rectangular Kirchhoff plate of length $a$ and width $b$ resting on a Winkler foundation as shown in Figure 1, was considered in this study. 


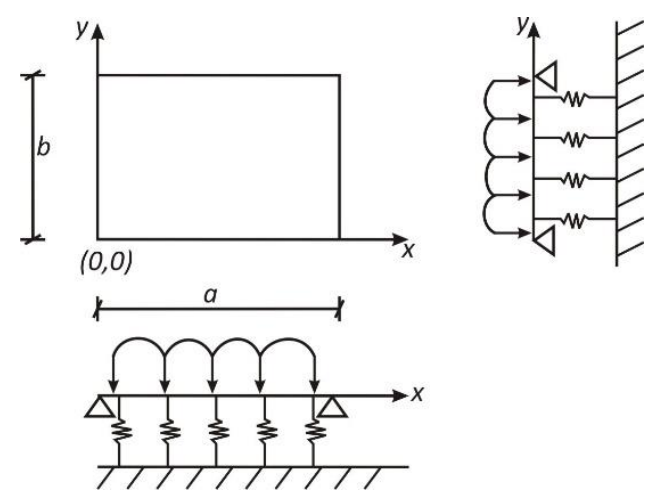

Figure 1. Rectangular Kirchhoff plate on Winkler foundation under arbitrary (general) load distribution

The boundary value problem at the Kirchhoff plate on Winkler foundation is given by the fourth order partial differential equation:

$$
D \nabla^{4} w(x, y)+k w(x, y)=q(x, y)
$$

or $\nabla^{4} w+\frac{k}{D} w=\frac{q}{D}$

for $0 \leq x \leq a 0 \leq y \leq b$

where $D=\frac{E h^{3}}{12\left(1-\mu^{2}\right)}$

$\nabla^{4}-\frac{\partial^{4}}{\partial x^{4}}+2 \frac{\partial^{4}}{\partial x^{2} \partial y^{2}}+\frac{\partial^{4}}{\partial y^{4}}=\nabla^{2} \nabla^{2}$

$D$ is the flexural rigidity of the plate material, $E$ is the Young's modulus of elasticity, $\mu$ is the Poisson's ratio, $h$ is the plate thickness, $k$ is the Winkler modulus of soil reaction, $w(x$, $y)$ is the transverse deflection of the plate's middle surface, $q(x$, $y$ ) is the distributed transverse load, and $x$ and $y$ are the inplane Cartesian coordinate variables.

For the case of Kirchhoff plate on Winkler foundation with simply supported edges at $x=0, x=a, y=0, y=b$, the geometric and force boundary conditions are:

at $x=0, x=a$,

$w(x=0, y)=0=w(x=a, y)$

$\frac{\partial^{2} w}{\partial x^{2}}(x=0, y)=0=\frac{\partial^{2} w}{\partial x^{2}}(x=a, y)$

at $y=0, y=b$,

$w(x, y=0)=0=w(x, y=b)$

$\frac{\partial^{2} w}{\partial y^{2}}(x, y=0)=0=\frac{\partial^{2} w}{\partial y^{2}}(x, y=b)$

\section{METHODOLOGY}

The finite Fourier sine transform method is an integral transformation technique for solving boundary value problems (BVP) of mathematical physics, introduced by Doetsch [15]. The method has been subsequently further developed by Kneitz [16], Strandhagen [17], Roettinger [18] and Brown [19]. The general principle of the method is to seek to simplify the BVP by elimination of partial derivatives with respect to the independent variables, thus yielding simplifications to the original problem. Thus partial differential equation (PDEs) expressed in terms of two independent coordinate variables are transformed by application of single finite Fourier sine transformation to ordinary differential equations (ODEs) and to algebraic equations by use of double finite Fourier sine transformation. Similarly, ODEs are transformed by single finite Fourier sine transform method to algebraic equations.

The finite Fourier sine transform of a function $w(x)$ of the independent variable $x$ is defined as the integral transformation:

$S_{m}=S(w(x))=\int_{0}^{l} w(x) \sin \frac{m \pi x}{l} d x$

where $m=1,2,3, \ldots ; 0 \leq x \leq l$.

The finite Fourier sine transform of the derivatives of $w(x)$ are obtained using the technique of integration by parts, in terms of the finite Fourier sine transform of the function $w(x)$. Similarly, for a function $w(x, y)$ of two independent variables $x$ and $y$, the finite Fourier sine transform, also called the double finite Fourier sine transform is defined by the integral transformation.

$S_{m n}=S\left(w(x, y)=W(m, n)=\int_{0}^{b} \int_{0}^{a} w(x, y) \sin \frac{m \pi x}{a} \sin \frac{n \pi y}{b} d x d y\right.$

where $m=1,2,3,4, \ldots ; n=1,2,3,4, \ldots ; 0 \leq x \leq a ; 0 \leq y \leq$ b

The kernel $K(m, n)$ of the Fourier sine transformation is given by:

$K(m, n)=\sin \frac{m \pi x}{a} \sin \frac{n \pi y}{b}$

By inversion, the unknown function $w(x, y)$ is recovered from the transform along the inversion formula:

$$
\begin{aligned}
& w(x, y)=\frac{4}{a b} \sum_{m=1}^{\infty} \sum_{n=1}^{\infty} W(m, n) \sin \frac{m \pi x}{a} \sin \frac{n \pi y}{b}, \quad m= \\
& 1,2,3,4, \ldots \infty ; \quad n=1,2,3,4, \ldots \infty
\end{aligned}
$$

For BVP with Drichlet boundary conditions, the finite Fourier sine transforms of the partial derivatives of $w(x, y)$ with respect to $x$ and $y$; as well as the mixed partial derivatives of $w(x, y)$ are found using integration by parts technique. Thus,

$$
\begin{aligned}
& \frac{\partial^{4} w}{\partial x^{4}}(x, y)=\frac{\partial^{4}}{\partial x^{4}} \int_{0}^{b} \int_{0}^{a} w(x, y) \sin \frac{m \pi x}{a} \sin \frac{n \pi y}{b} d x d y \\
& \frac{\partial^{4} w}{\partial x^{4}}=\left(\frac{m \pi}{a}\right)^{4} \int_{0}^{b} \int_{0}^{a} w(x, y) \sin \frac{m \pi x}{a} \sin \frac{n \pi y}{b} d x d y+\left(\frac{m \pi}{a}\right)^{3} \int_{0}^{b}\left(\left.(-1)^{m} w\right|_{x=a}-\left.w\right|_{x=0}\right) \sin \frac{n \pi y}{b} d y \\
& -\left(\frac{m \pi}{a}\right) \int_{0}^{b}\left(\left.(-1)^{m} \frac{\partial^{2} w}{\partial x^{2}}\right|_{x=a}-\left.\frac{\partial^{2} w}{\partial x^{2}}\right|_{x=0}\right) \sin \frac{n \pi y}{b} d y=\left(\frac{m \pi}{a}\right)^{4} W(m, n)
\end{aligned}
$$


Since we have Drichlet boundary conditions for simply supported Kirchhoff plate on Winkler foundation.

Similarly,

$\frac{\partial^{4} w(x, y)}{\partial x^{2} \partial y^{2}}=\frac{\partial^{4}}{\partial x^{2} \partial y^{2}} \int_{0}^{b} \int_{0}^{a} w(x, y) \sin \frac{m \pi x}{a} \sin \frac{n \pi y}{b} d x d y$

$\frac{\partial^{4} w(x, y)}{\partial x^{2} \partial y^{2}}=\left(\frac{m \pi}{a}\right)^{2}\left(\frac{n \pi}{b}\right)^{2} \int_{0}^{b} \int_{0}^{a} w(x, y) \sin \frac{m \pi x}{a} \sin \frac{n \pi y}{b} d x d y$

$\frac{\partial^{4} w(x, y)}{\partial x^{2} \partial y^{2}}=\left(\frac{m \pi}{a}\right)^{2}\left(\frac{n \pi}{b}\right)^{2} W(m, n)$

\section{RESULTS}

Application of the finite Fourier sine transform to the BVP, Equation (5) yields:

$\left.\int_{0}^{b} \int_{0}^{a}\left(\frac{\partial^{4} w}{\partial x^{4}}+2 \frac{\partial^{4} w}{\partial x^{2} \partial y^{2}}+\frac{\partial^{4} w}{\partial y^{4}}\right)+\frac{k}{D} w\right) \sin \frac{m \pi x}{a} \sin \frac{n \pi y}{b} d x d y=\int_{0}^{b} \int_{0}^{a} \frac{q(x, y)}{D} \sin \frac{m \pi x}{a} \sin \frac{n \pi y}{b} d x d y$

We employ the linearity property of the finite Fourier sine transform to obtain

$\int_{0}^{b} \int_{0}^{a} \frac{\partial^{4} w}{\partial x^{4}} \sin \frac{m \pi x}{a} \sin \frac{n \pi y}{b} d x d y+2 \int_{0}^{b} \int_{0}^{a} \frac{\partial^{4} w}{\partial x^{2} \partial y^{2}} \sin \frac{m \pi x}{a} \sin \frac{n \pi y}{b} d x d y+\int_{0}^{b} \int_{0}^{a} \frac{\partial^{4} w}{\partial y^{4}} \sin \frac{m \pi x}{a} \sin \frac{n \pi y}{b} d x d y$

$+\int_{0}^{b} \int_{0}^{a} \frac{k}{D} w(x, y) \sin \frac{m \pi x}{a} \sin \frac{n \pi y}{b} d x d y=\int_{0}^{b} \int_{0}^{a} \frac{q(x, y)}{D} \sin \frac{m \pi x}{a} \sin \frac{n \pi y}{b} d x d y$

Simplifying,

$\left(\frac{m \pi}{a}\right)^{4} W(m, n)+2\left(\frac{m \pi}{a}\right)^{2}\left(\frac{n \pi}{b}\right)^{2} W(m, n)+\left(\frac{n \pi}{b}\right)^{4} W(m, n)+\frac{k}{D} W(m, n)=\frac{q(m, n)}{D}$

where $W(m n)$ is given by Equation (14); and is the finite Fourier sine transform of the unknown function $w(x, y) ; q(m n)$ is the finite Fourier sine transform of the distributed transverse load, and is given by:

$q(m, n)=\int_{0}^{b} \int_{0}^{a} q(x, y) \sin \frac{m \pi x}{a} \sin \frac{n \pi y}{b} d x d y$

Solving,

$\left.\left(\left(\frac{m \pi}{a}\right)^{2}+\left(\frac{n \pi}{b}\right)^{2}\right)^{2}+\frac{k}{D}\right) W(m, n)=\frac{q(m, n)}{D}$

Thus,

$W(m, n)=\frac{q(m, n) / D}{\left(\left(\frac{m \pi}{a}\right)^{2}+\left(\frac{n \pi}{b}\right)^{2}\right)^{2}+\frac{k}{D}}$
$W(m, n)=\frac{q(m, n)}{D\left[\left(\left(\frac{m \pi}{a}\right)^{2}+\left(\frac{n \pi}{b}\right)^{2}\right)^{2}+\frac{k}{D}\right]}$

By inversion of the finite Fourier sine transform, the unknown deflection is obtained as follows:

$w(x, y)=\frac{4}{a b} \sum_{m}^{\infty} \sum_{n}^{\infty} \frac{q(m, n) \sin \frac{m \pi x}{a} \sin \frac{n \pi y}{b}}{D\left[\left(\left(\frac{m \pi}{a}\right)^{2}+\left(\frac{n \pi}{b}\right)^{2}\right)^{2}+\frac{k}{D}\right]}$

where $m=1,2,3,4, \ldots \infty ; n=1,2,3,4, \ldots \infty$.

\section{Bending moment distributions}

The bending moment distributions $M_{x x}, M_{y y}$ are obtained using the bending moment deflection equations. Thus,

$$
\begin{aligned}
& M_{x x}=-D\left(\frac{\partial^{2} w}{\partial x^{2}}+\mu \frac{\partial^{2} w}{\partial y^{2}}\right) \\
& M_{y y}=-D\left(\frac{\partial^{2} w}{\partial y^{2}}+\mu \frac{\partial^{2} w}{\partial x^{2}}\right) \\
& M_{x y}=-D(1-\mu) \frac{\partial^{2} w}{\partial x \partial y}
\end{aligned}
$$

By differentiation of Equation (29) and substitution into Equations $(30-32)$, we obtain:

$$
\begin{aligned}
& M_{x x}=\frac{4}{a b} \sum_{m}^{\infty} \sum_{n}^{\infty} \frac{\left(\left(\frac{m \pi}{a}\right)^{2}+\mu\left(\frac{n \pi}{b}\right)^{2}\right) q(m, n) \sin \frac{m \pi x}{a} \sin \frac{n \pi y}{b}}{\left(\left(\frac{m \pi}{a}\right)^{2}+\left(\frac{n \pi}{b}\right)^{2}\right)^{2}+\frac{k}{D}} \\
& M_{y y}=\frac{4}{a b} \sum_{m}^{\infty} \sum_{n}^{\infty} \frac{\left(\left(\frac{n \pi}{b}\right)^{2}+\mu\left(\frac{m \pi}{a}\right)^{2}\right) q(m, n) \sin \frac{m \pi x}{a} \sin \frac{n \pi y}{b}}{\left(\left(\frac{m \pi}{a}\right)^{2}+\left(\frac{n \pi}{b}\right)^{2}\right)^{2}+\frac{k}{D}} \\
& M_{x y}=\frac{-4}{a b}(1-\mu) \sum_{m}^{\infty} \sum_{n}^{\infty} \frac{\left(\frac{m \pi}{a} \frac{n \pi}{b}\right) q_{m n} \sin \frac{m \pi x}{a} \sin \frac{n \pi y}{b}}{\left[\left(\left(\frac{m \pi}{a}\right)^{2}+\left(\frac{n \pi}{b}\right)^{2}\right)^{2}+\frac{k}{D}\right]}
\end{aligned}
$$

\section{Finite Fourier sine transform solution for point load $P_{1}$ at $\left(x_{1}, y_{1}\right)\left(0 \leq x_{1} \leq a, 0 \leq y_{1} \leq b\right)$}

The flexural behaviour of Kirchhoff plate on elastic foundation under point load $P_{1}$ acting at a point $\left(x_{1}, y_{1}\right)$ on the plate region is obtained using Dirac delta function theory in the general solutions obtained to have:

$$
q(m, n)=\frac{4}{a b} \int_{0}^{b} \int_{0}^{a} P_{1} \delta\left(x=x_{1}, y=y_{1}\right) \sin \frac{m \pi x}{a} \sin \frac{n \pi y}{b} d x d y
$$


$q(m, n)=\frac{4 P_{1}}{a b} \sin \frac{m \pi x_{1}}{a} \sin \frac{n \pi y_{1}}{b}$

$w(x, y)=\frac{4 P_{1}}{a b D} \sum_{m}^{\infty} \sum_{n}^{\infty} \frac{\sin \frac{m \pi x_{1}}{a} \sin \frac{n \pi y_{1}}{b} \sin \frac{m \pi x}{a} \sin \frac{n \pi y}{b}}{\left[\left(\left(\frac{m \pi}{a}\right)^{2}+\left(\frac{n \pi}{b}\right)^{2}\right)^{2}+\frac{k}{D}\right]}$

$M_{x x}=\frac{4 P_{1}}{a b} \sum_{m}^{\infty} \sum_{n}^{\infty} \frac{\left(\left(\frac{m \pi}{a}\right)^{2}+\mu\left(\frac{n \pi}{b}\right)^{2}\right) \sin \frac{m \pi x_{1}}{a} \sin \frac{n \pi y_{1}}{b} \sin \frac{m \pi x}{a} \sin \frac{n \pi y}{b}}{\left[\left(\left(\frac{m \pi}{a}\right)^{2}+\left(\frac{n \pi}{b}\right)^{2}\right)^{2}+\frac{k}{D}\right]}$

$M_{y y}=\frac{4 P_{1}}{a b} \sum_{m}^{\infty} \sum_{n}^{\infty} \frac{\left(\left(\frac{n \pi}{b}\right)^{2}+\mu\left(\frac{m \pi}{a}\right)^{2} \sin \frac{m \pi x_{1}}{a} \sin \frac{n \pi y_{1}}{b} \sin \frac{m \pi x}{a} \sin \frac{n \pi y}{b}\right)}{\left(\left(\frac{m \pi}{a}\right)^{2}+\left(\frac{n \pi}{b}\right)^{2}\right)^{2}+\frac{k}{D}}$

where $m=1,3,5,7, \ldots ; n=1,3,5,7, \ldots$.

For a point load $P_{1}$ acting at the centre of the Kirchhoff plate on Winkler foundation $x_{1}=a / 2, y_{1}=b / 2$, and the maximum deflection and bending moments would occur at the plate centre, and are obtained as follows:

$w_{c}=w_{\max }=\frac{4 P_{1}}{a b D} \sum_{m}^{\infty} \sum_{n}^{\infty} \frac{\sin ^{2} \frac{m \pi}{2} \sin ^{2} \frac{n \pi}{2}}{\left(\left(\frac{m \pi}{a}\right)^{2}+\left(\frac{n \pi}{b}\right)^{2}\right)^{2}+\frac{k}{D}}$

$M_{x x_{\max }}=\frac{4 P_{1}}{a b} \sum_{m}^{\infty} \sum_{n}^{\infty} \frac{\left(\left(\frac{m \pi}{a}\right)^{2}+\mu\left(\frac{n \pi}{b}\right)^{2}\right) \sin ^{2} \frac{m \pi}{2} \sin ^{2} \frac{n \pi}{2}}{\left(\left(\frac{m \pi}{a}\right)^{2}+\left(\frac{n \pi}{b}\right)^{2}\right)^{2}+\frac{k}{D}}$

$M_{y y_{\max }}=\frac{4 P_{1}}{a b} \sum_{m}^{\infty} \sum_{n}^{\infty} \frac{\left(\left(\frac{n \pi}{b}\right)^{2}+\mu\left(\frac{m \pi}{a}\right)^{2}\right) \sin ^{2} \frac{m \pi}{2} \sin ^{2} \frac{n \pi}{2}}{\left(\left(\frac{m \pi}{a}\right)^{2}+\left(\frac{n \pi}{b}\right)^{2}\right)^{2}+\frac{k}{D}}$

For simply supported square Kirchhoff plate resting on Winkler foundation under point load $P_{1}$ applied at the plate centre, $a=b$, and the maximum deflection and bending moments occur at the centre and are obtained as follows:

$w_{\max }=\frac{4 P_{1} a^{2}}{D} \sum_{m}^{\infty} \sum_{n}^{\infty} \frac{\sin ^{2} \frac{m \pi}{2} \sin ^{2} \frac{n \pi}{2}}{\left(\pi^{4}\left(m^{2}+n^{2}\right)^{2}+\frac{k a^{4}}{D}\right)}$

$M_{\max }=4 P_{1} \sum_{m}^{\infty} \sum_{n}^{\infty} \frac{\pi^{2}\left(m^{2}+\mu n^{2}\right) \sin ^{2} \frac{m \pi}{2} \sin ^{2} \frac{n \pi}{2}}{\pi^{4}\left(m^{2}+n^{2}\right)^{2}+\frac{k a^{4}}{D}}$

$M_{y y_{\max }}=M_{x x_{\max }}=M_{x x}\left(\frac{a}{2}, \frac{a}{2}\right)$
Finite Fourier sine transform solution for transverse sinusoidal load $q(x, y)=q_{1} \sin \frac{\pi x}{a} \sin \frac{\pi y}{b}$

Here,

$q(m, n)=\int_{0}^{b} \int_{0}^{a} q_{1} \sin \frac{\pi x}{a} \sin \frac{\pi y}{b} \sin \frac{m \pi x}{a} \sin \frac{n \pi y}{b} d x d y$

$q(m, n)=q_{1} \int_{0}^{a} \sin \frac{\pi x}{a} \sin \frac{m \pi x}{a} d x \int_{0}^{b} \sin \frac{\pi y}{b} \sin \frac{m \pi y}{b} d y$

$q(m, n)=q_{1} \frac{a}{2} \frac{b}{2}=q_{1} \frac{a b}{4}$

where $m=1, n=1$.

$q(m, n)=0$ if $m \neq 1, n \neq 1(50)$

Then,

$$
\begin{aligned}
& w(x, y)=\frac{4}{a b} \sum_{m=1} \sum_{n=1} \frac{q_{1} \frac{a b}{4} \sin \frac{m \pi x}{a} \sin \frac{n \pi y}{b}}{D\left(\left(\left(\frac{m \pi}{a}\right)^{2}+\left(\frac{n \pi}{b}\right)^{2}\right)^{2}+\frac{k}{D}\right)} \\
& w(x, y)=\frac{q_{1} \sin \frac{\pi x}{a} \sin \frac{\pi y}{b}}{D\left[\left(\left(\frac{\pi}{a}\right)^{2}+\left(\frac{\pi}{b}\right)^{2}\right)^{2}+\frac{k}{D}\right]} \\
& M_{x x}=\frac{\left(\left(\frac{\pi}{a}\right)^{2}+\mu\left(\frac{\pi}{b}\right)^{2}\right) q_{1} \sin \frac{\pi x}{a} \sin \frac{\pi y}{b}}{\left(\left(\frac{\pi}{a}\right)^{2}+\left(\frac{\pi}{b}\right)^{2}\right)^{2}+\frac{k}{D}}
\end{aligned}
$$

$M_{y y}=\frac{\left(\left(\frac{\pi}{b}\right)^{2}+\mu\left(\frac{\pi}{a}\right)^{2}\right) q_{1} \sin \frac{\pi x}{a} \sin \frac{\pi y}{b}}{\left(\left(\frac{\pi}{a}\right)^{2}+\left(\frac{\pi}{b}\right)^{2}\right)^{2}+\frac{k}{D}}$

The maximum values of deflection and bending moments are found at the plate centre(s) as follows:

$$
\begin{aligned}
& w_{\text {max }}=w\left(\frac{a}{2}, \frac{b}{2}\right)=\frac{q_{1} \sin ^{2} \frac{\pi}{2}}{D\left[\left(\left(\frac{\pi}{a}\right)^{2}+\left(\frac{\pi}{b}\right)^{2}\right)^{2}+\frac{k}{D}\right]} \\
& M_{x x_{\max }}=M_{x x}\left(\frac{a}{2}, \frac{b}{2}\right)=\frac{\left(\left(\frac{\pi}{a}\right)^{2}+\mu\left(\frac{\pi}{b}\right)^{2}\right) q_{1} \sin ^{2} \frac{\pi}{2}}{\left[\left(\left(\frac{\pi}{a}\right)^{2}+\left(\frac{\pi}{b}\right)^{2}\right)^{2}+\frac{k}{D}\right]}
\end{aligned}
$$

where $m=1,3,5,7, \ldots ; n=1,3,5,7, \ldots$. 
$M_{y y_{\max }}=M_{y y}\left(\frac{a}{2}, \frac{b}{2}\right)=\frac{\left(\left(\frac{\pi}{b}\right)^{2}+\mu\left(\frac{\pi}{a}\right)^{2}\right) q_{1} \sin ^{2} \frac{\pi}{2}}{\left[\left(\left(\frac{\pi}{a}\right)^{2}+\left(\frac{\pi}{b}\right)^{2}\right)^{2}+\frac{k}{D}\right]}$

For simply supported plates on Winkler foundations, $a=b$, and we obtain the deflections and bending moments as follows:

$$
\begin{aligned}
& w(x, y)=\frac{q_{1} \sin \frac{\pi x}{a} \sin \frac{\pi y}{a}}{D\left[\left(\left(\frac{\pi}{a}\right)^{2}+\left(\frac{\pi}{a}\right)^{2}\right)^{2}+\frac{k}{D}\right]} \\
& w(x, y)=\frac{q_{1} \sin \frac{\pi x}{a} \sin \frac{\pi y}{a}}{D\left(\frac{4 \pi^{4}}{a^{4}}+\frac{k}{D}\right)} \\
& w(x, y)=\frac{q_{1} a^{4}}{D} \frac{\sin \frac{\pi x}{a} \sin \frac{\pi y}{a}}{\left(4 \pi^{4}+\frac{k a^{4}}{D}\right)}
\end{aligned}
$$$$
w\left(\frac{a}{2}, \frac{a}{2}\right)=\frac{q_{1} a^{4}}{D} \frac{\sin ^{2} \pi / 2}{\left(4 \pi^{4}+\frac{k a^{4}}{D}\right)}
$$$$
w_{\max }=\frac{1}{\left(4 \pi^{4}+\frac{k a^{4}}{D}\right)} \frac{q_{1} a^{4}}{D}
$$

Similarly, the finite Fourier sine transform solutions for bending moment expressions for simply supported square Kirchhoff plates on Winkler foundations are obtained for sinusoidal transverse load as:

$$
M_{x x}=\frac{\frac{\pi^{2}}{a^{2}}(1+\mu) q_{1} \sin \frac{\pi x}{a} \sin \frac{\pi y}{a}}{\frac{4 \pi^{4}}{a^{4}}+\frac{k}{D}}
$$

$$
M_{x x}=\frac{\pi^{2} a^{2}(1+\mu) q_{1} \sin \frac{\pi x}{a} \sin \frac{\pi y}{a}}{4 \pi^{4}+\frac{k a^{4}}{D}}
$$

$$
M_{x x}=\left(\frac{\pi^{2}(1+\mu)}{4 \pi^{4}+\frac{k a^{4}}{D}}\right) q_{1} a^{2} \sin \frac{\pi x}{a} \sin \frac{\pi y}{a}
$$

$$
\begin{aligned}
& M_{x x_{\max }}=M_{x x}\left(\frac{a}{2}, \frac{a}{2}\right)=\left(\frac{\pi^{2}(1+\mu)}{4 \pi^{4}+\frac{k a^{4}}{D}}\right) q_{1} a^{2} \sin ^{2}\left(\frac{\pi}{2}\right) \\
& M_{x x_{\max }}=\left(\frac{\pi^{2}(1+\mu)}{4 \pi^{4}+\frac{k a^{4}}{D}}\right) q_{1} a^{2}=M_{y y_{\max }}
\end{aligned}
$$

The twisting moment is obtained for rectangular thin plate on Winkler foundation as:

$$
\begin{aligned}
& M_{x y}=\frac{-D(1-\mu) q_{1} \frac{\pi}{a} \frac{\pi}{b} \cos \frac{\pi x}{a} \cos \frac{\pi y}{b}}{D\left[\left(\left(\frac{\pi}{a}\right)^{2}+\left(\frac{\pi}{b}\right)^{2}\right)^{2}+\frac{k}{D}\right]} \\
& M_{x y}=\frac{-(1-\mu) \pi^{2} q_{1}}{a b} \frac{\cos \frac{\pi x}{a} \cos \frac{\pi y}{b}}{\left[\pi^{4}\left(\frac{1}{a^{2}}+\frac{1}{b^{2}}\right)^{2}+\frac{k}{D}\right]}
\end{aligned}
$$

For square plates,

$$
\begin{aligned}
& M_{x y}=\frac{-(1-\mu) \pi^{2} q_{1}}{a^{2}} \frac{\cos \frac{\pi x}{a} \cos \frac{\pi y}{a}}{\left(\pi^{4}\left(\frac{2}{a^{2}}\right)^{2}+\frac{k}{D}\right)} \\
& M_{x y}=\frac{-(1-\mu) q_{1} a^{2} \pi^{2} \cos \frac{\pi x}{a} \cos \frac{\pi y}{a}}{4 \pi^{4}+\frac{k a^{4}}{D}}
\end{aligned}
$$

The maximum twisting moment is obtained at $x=0, y=0$, and is given by:

$$
M_{x y_{\max }}=-\frac{(1-\mu) \pi^{2} q_{1} a^{2}}{\left(4 \pi^{4}+\frac{k a^{4}}{D}\right)}
$$

Finite Fourier sine transform solutions for uniformly distributed load $q(x, y)=q_{0}$

For uniformly distributed load over the entire plate surface,

$$
\begin{aligned}
& q(m, n)=\int_{0}^{b} \int_{0}^{a} q_{0} \sin \frac{m \pi x}{a} \sin \frac{n \pi y}{b} d x d y \\
& q(m, n)=\frac{4 q_{0} a b}{m n \pi^{2}}
\end{aligned}
$$


Then, the deflections and bending moment expressions become:

$$
w(x, y)=\frac{4}{a b} \sum_{m}^{\infty} \sum_{n}^{\infty} \frac{\frac{4 q_{0} a b}{m n \pi^{2}} \sin \frac{m \pi x}{a} \sin \frac{n \pi y}{b}}{D\left[\left(\left(\frac{m \pi}{a}\right)^{2}+\left(\frac{n \pi}{b}\right)^{2}\right)^{2}+\frac{k}{D}\right]}
$$

$w(x, y)=\frac{16 q_{0}}{\pi^{2} D} \sum_{m}^{\infty} \sum_{n}^{\infty} \frac{\sin \frac{m \pi x}{a} \sin \frac{n \pi y}{b}}{m n\left[\pi^{4}\left(\left(\frac{m}{a}\right)^{2}+\left(\frac{n}{b}\right)^{2}\right)^{2}+\frac{k}{D}\right]}$

$m=1,3,5,7 \ldots ; n=1,3,5,7 .$.

$M_{x x}=\frac{16 q_{0}}{\pi^{2}} \sum_{m}^{\infty} \sum_{n}^{\infty} \frac{\left(\left(\frac{m \pi}{a}\right)^{2}+\mu\left(\frac{n \pi}{b}\right)^{2}\right) \sin \frac{m \pi x}{a} \sin \frac{n \pi y}{b}}{m n\left[\left(\left(\frac{m \pi}{a}\right)^{2}+\left(\frac{n \pi}{b}\right)^{2}\right)^{2}+\frac{k}{D}\right]}$

$M_{y y}=\frac{16 q_{0}}{\pi^{2}} \sum_{m}^{\infty} \sum_{n}^{\infty} \frac{\left(\left(\frac{n \pi}{b}\right)^{2}+\mu\left(\frac{m \pi}{a}\right)^{2}\right) \sin \frac{m \pi x}{a} \sin \frac{n \pi y}{b}}{m n\left[\left(\left(\frac{m \pi}{a}\right)^{2}+\left(\frac{n \pi}{b}\right)^{2}\right)^{2}+\frac{k}{D}\right]}$

The maximum deflection and bending moment, expectedly are found at the centre, as:

$w_{\max }=\frac{16 q_{0}}{D \pi^{2}} \sum_{m}^{\infty} \sum_{n}^{\infty} \frac{\sin \frac{m \pi}{2} \sin \frac{n \pi}{2}}{m n\left(\pi^{4}\left(\frac{m^{2}}{a^{2}}+\frac{n^{2}}{b^{2}}\right)^{2}+\frac{k}{D}\right)}$

$m=1,3,5,7 \ldots, \infty ; \quad n=1,3,5,7 \ldots, \infty$

$M_{x x \max }=\frac{16 q_{0}}{\pi^{2}} \sum_{m}^{\infty} \sum_{n}^{\infty} \frac{\left(\left(\frac{m \pi}{a}\right)^{2}+\mu\left(\frac{n \pi}{b}\right)^{2}\right) \sin \frac{m \pi}{2} \sin \frac{n \pi}{2}}{m n\left(\left(\left(\frac{m \pi}{a}\right)^{2}+\left(\frac{n \pi}{b}\right)^{2}\right)^{2}+\frac{k}{D}\right)}$

$M_{x x \max }=16 q_{0} a^{2} \sum_{m}^{\infty} \sum_{n}^{\infty} \frac{\left(m^{2}+\mu(n r)^{2}\right) \sin \left(\frac{m \pi}{2}\right) \sin \left(\frac{n \pi}{2}\right)}{m n\left[\pi^{4}\left(m^{2}+(n r)^{2}\right)^{2}+\frac{k a^{4}}{D}\right]}$

where $r=a / b, r$ is the plate aspect ratio.

Similarly,

$M_{y y \max }=16 q_{0} a^{2} \sum_{m}^{\infty} \sum_{n}^{\infty} \frac{\left(n r^{2}+\mu m^{2}\right) \sin \frac{m \pi}{2} \sin \frac{n \pi}{2}}{m n\left[\left(m^{2}+(n r)^{2}\right)^{2} \pi^{4}+\frac{k a^{4}}{D}\right]}$

For simply supported square Kirchhoff plate on Winkler foundation,

$$
\begin{aligned}
& w_{\text {max }}=\frac{16 q_{0} a^{4}}{D} \sum_{m}^{\infty} \sum_{n}^{\infty} \frac{\sin \frac{m \pi}{2} \sin \frac{n \pi}{2}}{m n\left(\left(m^{2}+n^{2}\right)^{2} \pi^{4}+\frac{k a^{4}}{D}\right)} \\
& M_{x x \max }=M_{y y \max }=16 q_{0} a^{2} \sum_{m}^{\infty} \sum_{n}^{\infty} \frac{\left(m^{2}+\mu n^{2}\right) \sin \frac{m \pi}{2} \sin \frac{n \pi}{2}}{m n\left(\left(m^{2}+n^{2}\right)^{2} \pi^{4}+\frac{k a^{4}}{D}\right)}
\end{aligned}
$$

The twisting moments $M_{x y}$ are obtained as:

$$
M_{x y}=\frac{-16 q_{0}(1-\mu) a^{3}}{b} \sum_{m}^{\infty} \sum_{n}^{\infty} \frac{1}{\pi^{4}\left(m^{2}+n^{2} r^{2}\right)^{2}+\frac{k a^{4}}{D}}
$$

For square plates, $r=1$,

$$
M_{x y}=-16 q_{0}(1-\mu) a^{2} \sum_{m}^{\infty} \sum_{n}^{\infty} \frac{1}{\left(\pi^{4}\left(m^{2}+n^{2}\right)^{2}+\frac{k a^{4}}{D}\right)}
$$

Finite Fourier Sine Transform Solutions for Linearly Distributed Load $q(x, y)=q_{0} x / a$

\section{$(0 \leq x \leq a, 0 \leq y \leq b)$}

For linearly distributed load over the entire plate surface,

$$
\begin{aligned}
& q_{m n}=\int_{0}^{b} \int_{0}^{a} q_{0} \frac{x}{a} \sin \frac{m \pi x}{a} \sin \frac{n \pi y}{b} d x d y \\
& q_{m n}=\frac{8 q_{0}}{m n} \frac{\cos m \pi}{\pi^{2}} \cdot \frac{a b}{4}=\frac{2 q_{0} a b \cos m \pi}{m n \pi^{2}} \\
& m=1,3,5,7 \ldots ; \quad n=1,3,5,7 \ldots
\end{aligned}
$$

The deflection field (function) becomes:

$$
w(x, y)=\left(\frac{4}{a b}\right) \frac{2 q_{0} a b}{\pi^{2} D} \sum_{m}^{\infty} \sum_{n}^{\infty} \frac{\cos m \pi \sin \frac{m \pi x}{a} \sin \frac{n \pi y}{b}}{m n\left[\left(\left(\frac{m \pi}{a}\right)^{2}+\left(\frac{n \pi}{b}\right)^{2}\right)^{2}+\frac{k}{D}\right]}
$$

$$
w=\frac{8 q_{0} a^{4}}{\pi^{2} D} \sum_{m}^{\infty} \sum_{n}^{\infty} \frac{\cos m \pi \sin \frac{m \pi x}{a} \sin \frac{n \pi y}{b}}{m n\left[\pi^{4}\left(m^{2}+(n r)^{2}\right)^{2}+\frac{k a^{4}}{D}\right]}
$$

At the plate centre, $x=a / 2, y=b / 2$,

$$
w_{\text {max }}=w(a / 2, b / 2)=\frac{8 q_{0} a^{4}}{\pi^{2} D} \sum_{m}^{\infty} \sum_{n}^{\infty} \frac{\cos m \pi \sin \frac{m \pi}{2} \sin \frac{n \pi}{2}}{m n\left[\pi^{4}\left(m^{2}+n^{2} r^{2}\right)^{2}+\frac{k a^{4}}{D}\right]}
$$

The bending moments are obtained from the bending moment curvature (bending moment displacement) relations as: 
$M_{x x}=8 q_{0} a^{2} \sum_{m}^{\infty} \sum_{n}^{\infty} \frac{\left(m^{2}+\mu n^{2} r^{2}\right) \cos m \pi \sin \frac{m \pi x}{a} \sin \frac{n \pi y}{b}}{\left(\pi^{4}\left(m^{2}+(n r)^{2}\right)^{2}+\frac{k a^{4}}{D}\right) m n}$

$M_{y y}=8 q_{0} a^{2} \sum_{m}^{\infty} \sum_{n}^{\infty} \frac{\left(n^{2} r^{2}+\mu m^{2}\right) \cos m \pi \sin \frac{m \pi x}{a} \sin \frac{n \pi y}{b}}{\left(\pi^{4}\left(m^{2}+(n r)^{2}\right)^{2}+\frac{k a^{4}}{D}\right) m n}$

Bending moments at the plate centre are given by:

$M_{x x}\left(\frac{a}{2}, \frac{b}{2}\right)=8 q_{0} a^{2} \sum_{m}^{\infty} \sum_{n}^{\infty} \frac{\left(m^{2}+\mu n^{2} r^{2}\right) \cos m \pi \sin \frac{m \pi}{2} \sin \frac{n \pi}{2}}{\left(\pi^{4}\left(m^{2}+(n r)^{2}\right)^{2}+\frac{k a^{4}}{D}\right) m n}$

$M_{y y}\left(\frac{a}{2}, \frac{b}{2}\right)=8 q_{0} a^{2} \sum_{m}^{\infty} \sum_{n}^{\infty} \frac{\left(n^{2} r^{2}+\mu m^{2}\right) \cos m \pi \sin \frac{m \pi}{2} \sin \frac{n \pi}{2}}{\left(\pi^{4}\left(m^{2}+(n r)^{2}\right)^{2}+\frac{k a^{4}}{D}\right) m n}$

For square Kirchhoff plates on Winkler foundation, $r=1$, and

$w_{\max }=\frac{8 q_{0} a^{4}}{\pi^{2} D} \sum_{m}^{\infty} \sum_{n}^{\infty} \frac{\cos m \pi \sin \frac{m \pi}{2} \sin \frac{n \pi}{2}}{m n\left(\pi^{4}\left(m^{2}+n^{2}\right)^{2}+\frac{k a^{4}}{D}\right)}$
$M_{x x c}=8 q_{0} a^{2} \sum_{m}^{\infty} \sum_{n}^{\infty} \frac{\left(m^{2}+\mu n^{2}\right) \cos m \pi \sin \frac{m \pi}{2} \sin \frac{n \pi}{2}}{m n\left(\pi^{4}\left(m^{2}+n^{2}\right)^{2}+\frac{k a^{4}}{D}\right)}$

$M_{y y c}=8 q_{0} a^{2} \sum_{m}^{\infty} \sum_{n}^{\infty} \frac{\left(n^{2} r^{2}+\mu m^{2}\right) \cos m \pi \sin \frac{m \pi}{2} \sin \frac{n \pi}{2}}{m n\left(\pi^{4}\left(m^{2}+n^{2}\right)^{2}+\frac{k a^{4}}{D}\right)}$

The Finite Fourier sine transform solutions for the maximum deflection and maximum bending moments which occur at the plate centre $(x=a / 2, y=b / 2)$ for square simply supported Kirchhoff plates resting on Winkler foundations for values of the dimensionless Winkler parameter $K=\left(k a^{4} / D\right)^{1 / 4}$ where $K=0, K=1, K=3$, and $K=5$ were determined and presented in Table 1 for the case of uniformly distributed transverse load of intensity $q_{0}$ over the plate region. Similarly, the finite Fourier sine transform solutions for the maximum deflection and bending moments for square simply supported Kirchhoff plates resting on Winkler foundations for values of the dimensionless Winkler parameter $K$ given by $K$ $=0, K=1, K=3, K=5$ and $K=7$ for the case of sinusoidal load distribution over the plate domain were computed and shown in Table 2.

Table 1. Finite Fourier sine transform solutions for maximum deflection and bending moments for simply supported square Kirchhoff plate on Winkler foundation under uniform load, $\mu=0.30$

\begin{tabular}{|c|c|c|c|c|c|}
\hline$K$ & $K^{2}$ & $w_{\max }\left(\times 10^{-3} \frac{q a^{4}}{D}\right)$ & $M_{x x}\left(\times 10^{-2} q a^{2}\right)$ & $M_{y y}\left(\times 10^{-2} q a^{2}\right)$ & $M_{x y}\left(\times 10^{-2} q a^{2}\right)$ \\
\hline 0 & 0 & 4.062 & 4.790 & 4.790 & \\
\hline 1 & 1 & 4.053 & 4.809 & 4.809 & 2.943 \\
\hline 3 & 81 & 3.348 & 3.910 & 3910 & 2.456 \\
\hline 5 & 625 & 1.507 & 1.575 & 1.575 & 1.181 \\
\hline
\end{tabular}

Table 2. Finite Fourier sine transform solutions for maximum deflection and bending moments in simply supported square Kirchhoff plate on Winkler foundation under transverse sinusoidal load $q(x, y)=q_{0} \sin \pi x / a \sin \pi y / b \quad(\mu=0.30)$

\begin{tabular}{|c|c|c|c|c|c|}
\hline$K$ & $K^{4}$ & $w_{\max }\left(\times 10^{-3} \frac{q_{0} a^{4}}{D}\right)$ & $M_{x x}\left(\times 10^{-2} q a^{2}\right)$ & $M_{y y}\left(\times 10^{-2} q a^{2}\right)$ & $M_{x y}\left(\times 10^{-2} q a^{2}\right)$ \\
\hline 0 & 0 & 2.566495 & 3.29294 & 3.29294 & 1.797 \\
\hline 1 & 1 & 2.559925 & 3.28451 & 3.28451 & 1.792 \\
\hline 3 & 81 & 2.124782 & 2.7262 & 2.7262 & 1.487 \\
\hline 5 & 625 & 0.985574 & 1.26454 & 1.26454 & 0.06899 \\
\hline 7 & 240 & 0.358341 & 0.45977 & 0.45977 & 0.025084 \\
\hline
\end{tabular}

\section{DISCUSSION}

The finite Fourier sine transform method has been successfully implemented in this work to solve the flexural problems of simply supported rectangular Kirchhoff plates resting on Winkler foundations where the plate is under transverse distributed loads. The problem was presented as a boundary value problem involving the fourth order partial differential equation given as Equation (5) subject to the boundary conditions in Equations $(9-12)$. Application of the finite Fourier sine transformation to the boundary value problem for the general case of arbitrary distribution of load yielded the solution for the deflection in the transform space as Equation (28). By inversion, the unknown deflection was obtained for any distribution of load as Equation (29). Bending and twisting moments were obtained using the moment 
displacement or moment curvature relations as Equation (3335).

Particular cases of transverse loads were considered, and solved for. The solutions for deflections and bending moments for the case of point load applied at any point on the plate domain were found as Equations $(38-40)$. It was observed that maximum deflections and bending moments occur at the plate centre for the case of point load applied at the centre, and their values were obtained as Equations $(41$ - 43) for rectangular plates, and Equation (44-46) for square plates on Winkler foundations.

The solutions for rectangular Kirchhoff plate an Winkler foundation under sinusoidal load were found as Equation (52 - 54). The maximum values of the deflection and bending moments were found to occur at the plate centre, and were found as Equations (55 - 57) for rectangular plates and Equations (61), and (67) for square plate on Winkler foundation. Twisting moment expressions were found for rectangular plate as Equation (69) and Equation (71) for square thin plate on Winkler foundation. The maximum twisting moment was found at a corner of the plate as Equation (72).

The solutions for uniform transverse load over the entire plate domain were obtained as Equation $(76-78)$. The maximum deflection and bending moments were found to occur at the plate centre and were determined as Equation (79 - 82) for rectangular plates, and Equation (83) and (84) for square Kirchhoff plate on Winkler foundation. The twisting moment for uniform load was found as Equation (85) and Equation (86) for square Kirchhoff plate on Winkler foundation.

The solutions for linearly distributed load were obtained as Equations (90), (92) and (93). Expressions for the deflection and bending moments at the plate centre were found, in this case, as Equations (91), (94) and (95) for rectangular plates, and Equation (96 - 98) for square plates on Winkler foundations. For the case of linearly distributed load on the plate, the maximum values of deflection and bending moments may not occur at the plate centre due to the non-symmetrical load distribution with respect to the plate centre.

The finite Fourier sine transform solutions obtained for simply supported square Kirchhoff plate on Winkler foundation for the case of uniformly distributed transverse load which is shown in Table 1 for various values of the dimensionless Winkler parameter $K$, show that the maximum deflections and bending and twisting moments at the plate centre decrease as the elastic stiffness of the Winkler foundation, characterised by the dimensionless Winkler parameter $K$ increases. Similarly, Table 2 shows that the maximum deflection, bending and twisting moments at the plate centre reduce with increase in the dimensionless Winkler parameter $K$.

It is further observed that the solutions obtained in all cases of load considered in this study were double sine series of infinite terms. The double sine series for deflections were rapidly convergent for sinusoidal, uniform and linearly distributed loads, but less rapidly convergent for point load. The series for bending moments were less rapidly convergent even for sinusoidal, uniform and linearly distributed loads. The rapidly convergent properties of the double series obtained for the deflections for distributed loads ensured that reasonably accurate results were obtained using a few terms of the double series for the deflections. More terms of the series for bending moments were needed for satisfactory convergent results.
It is further observed that the finite Fourier sine transform solutions obtained in this work for simply supported Kirchhoff plates on Winkler foundations gave analytical closed form solutions which were exactly the same as solutions obtained using a Navier double trigonometric series technique for the problem.

\section{CONCLUSIONS}

The following conclusions can be made from this study:

(i)The finite Fourier sine transform method transforms the boundary value problem (BVP) of the flexure of simply supported Kirchhoff plate resting on Winkler foundation and carrying transverse loads to an algebraic equation in terms of the transform variables $\mathrm{m}, \mathrm{n}$.

(ii) The finite Fourier sine transform transforms the boundary value problem of bending of simply supported Kirchhoff plate on Winkler foundation for the case of any load to an algebraic problem in the transform space variables.

(iii)The finite Fourier sine transform method yielded mathematically closed form solutions for the deflection, bending and twisting moments for the rectangular Kirchhoff plate on Winkler foundation with simply supported edges, and under transverse distributed load.

(iv)The analytical closed form solutions obtained using the method were exact solutions within the scope, limitations and fundamental assumptions of the classical Kirchhoff's small deformation thin plate theory and the Winkler foundation model used in formulating the BVP.

(v)The finite Fourier sine transform solutions are exactly identical with the solutions obtained using Navier's trigonometric series technique for the same problem.

(vi)The Winkler foundation has the effect of reducing the maximum deflections and bending moments at the centre of the plate for loads distributed symmetrically about the plate centre.

(vii)The Dirichilet boundary conditions associated with simply supported edges $\mathrm{x}=0, \mathrm{x}=\mathrm{a}, \mathrm{y}=0, \mathrm{y}=\mathrm{b}$ greatly simplified the applications of the finite Fourier sine transformation of the BVP.

(viii)Convergence of the double sine series expressions obtained for displacements were more rapid than those obtained for the bending and twisting moments.

(ix)Convergence of the double sine series expressions obtained for the case of point load on the plate was very slow due to the singularity property of the point load and its representation using many terms of the finite Fourier sine transformation.

\section{REFERENCES}

[1] Reissner E. (1944). On the theory of bending of elastic plates, Journal of Mathematics and Physics, Vol. 23, pp. $184-191$.

[2] Reissner E. (1945). The effect of shear deformation on the bending of elastic plates, Journal of Applied Mechanics, Vol. 12, pp. $69-75$

[3] Mindlin R.D. (1951). Influence of rotary inertia on flexural motion of isotropic, elastic plates, Journal of Applied Mechanics, Vol. 18, No. 1, pp. 31 - 38.

[4] Shimpi R.P. (2002). Refined plate theory and its variants, AIAA Journal, Vol. 40, pp. 137 -146. 
[5] Reddy J.N. (1984). A refined non-linear theory of plates with transverse shear deformation, International Journal of Solids and Structures, Vol. 20, pp. $881-896$.

[6] Mama B.O., Ike C.C., Onah H.N. Nwoji C.U. (2017). Analysis of rectangular Kirchhoff plate on Winkler foundation using finite Fourier sine transform method, IOSR Journal of Mathematics (IOSR JM), Vol. 13, No. 1, pp. 58-66. DOI: $10.9790 / 5728$ - 1301065866

[7] Caselunghe A., Eriksson J. (2012). Structural element App.roaches for Soil Structure Interaction MSc Thesis, Chalmar University of Technology Goterborg, Sweden.

[8] Rajpurohit V.K., Gore N.G., Sayagavi V.C. (2014). Analysis of structures supported on elastic foundation, International Journal of Engineering and Technology (IJEAT), Vol. 4, No. 1.

[9] Ghaitani M.M., Esmaieili H.A. Kolahchi R. (2014). Elastic foundation effect on bending behaviour of oil pipelines, International Conference on Civil, Biological and Environmental Engineering (CBEE), Istanbul, Turkey.

[10] Winkler E. (1867). Die Lehre vonder elastizitat und festigkeit Prague.

[11] Pasternak P.L. (1954). On a new method of analysis of an elastic foundation by means of two foundation constants (in Russian) Gosud Izd Lit po Stroitelstvu, Arkhitecture Moscow.
[12] Filonenko-Borodich M.M. (1945). A very simple model of an elastic foundation capable of spreading load, Shornik Moskovkovo Elecktro Instituta.

[13] Hetenyi M. (1946). Beams on elastic foundation: theory with applications in the fields of civil and mechanical engineering, The University of Michigan Press, Michigan.

[14] Kerr A.D. (1965). A study of a new foundation model, Acta Mechanica, Vol. 1, pp. 135 - 147.

[15] Doetsch G. (1935). Integration von differentialglei chungen vermittels der endlichen, Fourier Transformation Math Ann, Vol. 112, pp. 52 - 68 .

[16] Kneitz K. (1938). Lösung von Randwert problem bei systemem gewöhullicher Differentialglei-chungen vermittels der endlichen, Fourier Transformation Math Zeit, Vol. 44, pp. $266-291$.

[17] Strandhagen G. (1944). Use of sine transformation for non-simply supp.orted beams, Quart. Appl. Math 1, pp. $346-348$.

[18] Roettinger I. (1945). An operational approach to the solution of boundary value problems by generalised Fourier series, Bull American Math Soc., Vol. 51, p. 67.

[19] Brown H.K. (1944). Resolution of temperature problems by use of finite Fourier transformation, Bull American Math Soc., Vol. 50, pp. 376 - 385. 